\title{
A Note on Symmetric Properties of the Twisted $q$-Bernoulli Polynomials and the Twisted Generalized $q$-Bernoulli Polynomials
}

\author{
L.-C. Jang, ${ }^{1}$ H. Yi, ${ }^{2}$ K. Shivashankara, ${ }^{3}$ T. Kim, $^{4}$ Y. H. Kim, ${ }^{4}$ \\ and B. Lee ${ }^{5}$ \\ ${ }^{1}$ Department of Mathematics and Computer Science, KonKuk University, \\ Chungju 138-70, Republic of Korea \\ 2 Department of Mathematics, Kwangwoon University, Seoul 139-701, Republic of Korea \\ ${ }^{3}$ Department of Mathematics, Yuvaraja's College, University of Mysore, Mysore 570\# 005, India \\ ${ }^{4}$ Division of General Education-Mathematics, Kwangwoon University, Seoul 139-701, Republic of Korea \\ ${ }^{5}$ Department of Wireless Communications Engineering, Kwangwoon University, \\ Seoul 139-701, Republic of Korea \\ Correspondence should be addressed to H. Yi, hsyi@kw.ac.kr
}

Received 11 September 2009; Revised 14 April 2010; Accepted 31 May 2010

Academic Editor: Abdelkader Boucherif

Copyright (C 2010 L.-C. Jang et al. This is an open access article distributed under the Creative Commons Attribution License, which permits unrestricted use, distribution, and reproduction in any medium, provided the original work is properly cited.

We define the twisted $q$-Bernoulli polynomials and the twisted generalized $q$-Bernoulli polynomials attached to $x$ of higher order and investigate some symmetric properties of them. Furthermore, using these symmetric properties of them, we can obtain some relationships between twisted $q$ Bernoulli numbers and polynomials and between twisted generalized $q$-Bernoulli numbers and polynomials.

\section{Introduction}

Let $p$ be a fixed prime number. Throughout this paper $\mathbb{Z}_{p}, \mathbb{Q}_{p}$, and $\mathbb{C}_{p}$ will, respectively, denote the ring of $p$-adic rational integers, the field of $p$-adic rational numbers, and the completion of algebraic closure of $\mathbb{Q}_{p}$. Let $v_{p}$ be the normalized exponential valuation of $\mathbb{C}_{p}$ with $|p|_{p}=$ $p^{-v_{p}(p)}=p^{-1}$. When one talks of $q$-extension, $q$ is variously considered as an indeterminate, a complex number $q \in \mathbb{C}$, or a $p$-adic number $q \in \mathbb{C}_{p}$. If $q \in \mathbb{C}$, one normally assumes $|q|<1$. If $q \in \mathbb{C}_{p}$, then we assume $|q-1|_{p}<p^{-1 /(p-1)}$, so that $q^{x}=\exp (x \log q)$ for $|x|_{p} \leq 1$ (cf. [1-32]). 
For $N, d \in \mathbb{N}$, we set

$$
X=X_{d}=\frac{\lim _{\overleftarrow{N}} \mathbb{Z}}{d p^{N} \mathbb{Z}}, \quad X_{1}=\mathbb{Z}_{p}
$$

(see [1-13]). The Bernoulli numbers $B_{n}$ and polynomials $B_{n}(x)$ are defined by the generating function as

$$
\begin{gathered}
\frac{t}{e^{t}-1}=\sum_{n=0}^{\infty} B_{n} \frac{t^{n}}{n !}, \\
\frac{t}{e^{t}-1} e^{x t}=\sum_{n=0}^{\infty} B_{n}(x) \frac{t^{n}}{n !}
\end{gathered}
$$

(cf. $[17,18,21,24,26])$. Let $U D(X)$ be the set of uniformly differentiable functions on $X$. For $f \in \mathrm{UD}(X)$, the $p$-adic invariant integral on $\mathbb{Z}_{p}$ is defined as

$$
I(f)=\int_{X} f(x) d x=\lim _{N \rightarrow \infty} \frac{1}{d p^{N}} \sum_{x=0}^{d p^{N}-1} f(x) .
$$

Note that $\int_{X} f(x) d x=\int_{\mathbb{Z}_{p}} f(x) d x$ (see [27]). Let $f_{n}(x)$ be a translation with $f_{n}(x)=f(x+n)$. We note that

$$
I\left(f_{n}\right)=I(f)+\sum_{i=0}^{n-1} f^{\prime}(i)
$$

(cf. [1-32]). Kim [18] studied the symmetric properties of the $q$-Bernoulli numbers and polynomials as follows:

$$
\frac{t+\log q}{q e^{t}-1} e^{x t}=\sum_{n=0}^{\infty} B_{n}^{q}(x) \frac{t^{n}}{n !}
$$

In this paper, we define the twisted $q$-Bernoulli polynomials and the twisted generalized $q$-Bernoulli polynomials attached to $x$ of higher order and investigate some symmetric properties of them. Furthermore, using these symmetric properties of them, we can obtain some relationships between the twisted $q$-Bernoulli numbers and polynomials and between the twisted generalized $q$-Bernoulli numbers and polynomials attached to $x$ of higher order. 


\section{The Twisted $q$-Bernoulli Polynomials}

Let $C_{p^{\infty}}=\bigcup_{n \geq 1} C_{p^{n}}=\lim _{n \rightarrow \infty} C_{p^{n}}$ be the locally constant space, where $C_{p^{n}}=\left\{\xi \mid \xi^{p^{n}}=1\right\}$ is the cyclic group of order $p^{n}$. For $w \in C_{p^{\infty}}$, we denote the locally constant function by

$$
\phi_{w}: \mathbb{Z}_{p} \longrightarrow \mathbb{C}_{p}, \quad x \longmapsto w^{x}
$$

(cf. $[2,3,21,24])$. If we take $f(x)=\phi_{w}(x) q^{x} e^{t x}$, then

$$
\int_{\mathbb{Z}_{p}} e^{x t} w^{x} q^{x} d x=\frac{\log q+t}{w q e^{t}-1}
$$
follows:

Now we define the $q$-extension of twisted Bernoulli numbers and polynomials as

$$
\begin{aligned}
\frac{\log q+t}{w q e^{t}-1} & =\sum_{n=0}^{\infty} B_{n, w}^{q} \frac{t^{n}}{n !}, \\
\frac{\log q+t}{w q e^{t}-1} e^{t x} & =\sum_{n=0}^{\infty} B_{n, w}^{q}(x) \frac{t^{n}}{n !}
\end{aligned}
$$

(see [31]). From (1.5), (2.2), (2.3), and (2.4), we can derive

$$
\int_{\mathbb{Z}_{p}} w^{y} q^{y}(x+y)^{n} d y=B_{n, w}^{q}(x), \quad \int_{\mathbb{Z}_{p}} w^{y} q^{y} y^{n} d y=B_{n, w}^{q}
$$

By (1.5), we can see that

$$
\begin{aligned}
& \frac{1}{\log q+t}\left(\int_{\mathbb{Z}_{p}} w^{n+x} q^{n+x} e^{(n+x) t} d x-\int_{\mathbb{Z}_{p}} w^{x} q^{x} e^{x t} d x\right) \\
& =\frac{w^{n} q^{n} e^{n t}-1}{t+\log q} \int_{\mathbb{Z}_{p}} w^{x} q^{x} e^{x t} d x \\
& =\frac{w^{n} q^{n} e^{n t}-1}{w q e^{t}-1} \\
& =\sum_{i=0}^{n-1} w^{i} q^{i} e^{i t} \\
& =\sum_{k=0}^{\infty}\left(\sum_{i=0}^{n-1} i^{k} w^{i} q^{i}\right) \frac{t^{k}}{k !} .
\end{aligned}
$$


In (1.4), it is easy to show that

$$
\frac{1}{\log q+t}\left(\int_{\mathbb{Z}_{p}} w^{n+x} q^{n+x} e^{(n+x) t} d x-\int_{\mathbb{Z}_{p}} w^{x} q^{x} e^{x t} d x\right)=\frac{n \int_{\mathbb{Z}_{p}} w^{x} q^{x} e^{x t} d x}{\int_{\mathbb{Z}_{p}} w^{n x} q^{n x} e^{n x t} d x}
$$

For each integer $k \geq 0$, let

$$
S_{k, w}^{q}(n)=0^{k}+1^{k} w q+2^{k} w^{2} q^{2}+\cdots+n^{k} w^{n} q^{n} .
$$

From (2.6), (2.7), and (2.8), we derive

$$
\frac{1}{\log q+t}\left(\int_{\mathbb{Z}_{p}} w^{n+x} q^{n+x} e^{(n+x) t} d x-\int_{\mathbb{Z}_{p}} w^{x} q^{x} e^{x t} d x\right)=\frac{n \int_{\mathbb{Z}_{p}} w^{x} q^{x} e^{x t} d x}{\int_{\mathbb{Z}_{p}} w^{n x} q^{n x} e^{n x t} d x}=\sum_{k=0}^{\infty} S_{k, w}^{q}(n-1) \frac{t^{k}}{k !}
$$

From (2.9), we note that

$$
B_{k, w}^{q}(n)-B_{k, w}^{q}=k S_{k-1, w}^{q}(n-1)+\log q S_{k, w}^{q}(n-1),
$$

for all $k, n \in \mathbb{N}$. Let $u_{1}, u_{2} \in \mathbb{N}$ and $w \in C_{p^{\infty}}$; then we have

$$
\frac{\int_{\mathbb{Z}_{p}} w^{u_{1} x_{1}+u_{2} x_{2}} q^{u_{1} x_{1}+u_{2} x_{2}} e^{u_{1} x_{1}+u_{2} x_{2}} d x_{1} d x_{2}}{\int_{\mathbb{Z}_{p}} w^{u_{1} u_{2} x} q^{u_{1} u_{2} x} e^{u_{1} u_{2} x t} d x}=(t+\log q) \frac{w^{u_{1} u_{2}} q^{u_{1} u_{2}} e^{u_{1} t}-1}{w^{u_{2}} q^{u_{2}} e^{u_{2} t}-1} .
$$

By (2.9), we see that

$$
\frac{u_{1} \int_{\mathbb{Z}_{p}} w^{x} q^{x} e^{x t} d x}{\int_{\mathbb{Z}_{p}} w^{u_{1} x} q^{u_{1} x} e^{u_{1} x t} d x}=\sum_{l=0}^{\infty}\left(\sum_{k=0}^{u_{1}-1} k^{l} w^{k} q^{k}\right) \frac{t^{l}}{l !}=\sum_{l=0}^{\infty} S_{l, w}^{q}\left(u_{1}-1\right) \frac{t^{l}}{l !}
$$

Let $T_{w}\left(u_{1}, u_{2} ; x, t\right)$ be as follows:

$$
T_{w}\left(u_{1}, u_{2} ; x, t\right)=\frac{\int_{\mathbb{Z}_{p}} w^{u_{1} x_{1}+u_{2} x_{2}} q^{u_{1} x_{1}+u_{2} x_{2}} e^{\left(u_{1} x_{1}+u_{2} x_{2}+u_{1} u_{2} x\right) t} d x_{1} d x_{2}}{\int_{\mathbb{Z}_{p}} w^{u_{1} u_{2} x} q^{u_{1} u_{2} x} e^{u_{1} u_{2} x t} d x}
$$

Then we have

$$
T_{w}\left(u_{1}, u_{2} ; x, t\right)=\frac{(t+\log q) e^{u_{1} u_{2} t}\left(w^{u_{1} u_{2}} q^{u_{1} u_{2}} e^{u_{1} u_{2} t}-1\right)}{\left(w^{u_{1}} q^{u_{1}} e^{u_{1} t}-1\right)\left(w^{u_{2}} q^{u_{2}} e^{u_{2} t}-1\right)}
$$


From (2.13), we derive

$$
T_{w}\left(u_{1}, u_{2} ; x, t\right)=\left(\frac{1}{u_{1}} \int_{\mathbb{Z}_{p}} w^{u_{1} x_{1}} q^{u_{1} x_{1}} e^{u_{1}\left(x_{1}+u_{2} x\right) t} d x_{1}\right)\left(\frac{u_{1} \int_{\mathbb{Z}_{p}} w^{u_{2} x_{2}} q^{u_{2} x_{2}} e^{u_{2} x_{2} t}}{\int_{\mathbb{Z}_{p}} w^{u_{1} u_{2} x} q^{u_{1} u_{2} x} e^{u_{1} u_{2} x t} d x}\right) .
$$

By (2.4), (2.12), and (2.15), we can see that

$$
\begin{aligned}
T_{w}\left(u_{1}, u_{2} ; x, t\right) & =\frac{1}{u_{1}}\left(\sum_{i=0}^{\infty} B_{i, w^{u_{1}}}^{q^{u_{1}}}\left(u_{2} x\right) \frac{u_{1}^{i} t^{i}}{i !}\right)\left(\sum_{l=0}^{\infty} S_{l, w^{u_{2}}}^{q^{u_{2}}}\left(u_{1}-1\right) \frac{u_{2}^{l} t^{l}}{l !}\right) \\
& =\sum_{n=0}^{\infty}\left(\sum_{i=0}^{n}\left(\begin{array}{c}
n \\
i
\end{array}\right) B_{i, w^{u_{1}}}^{q^{u_{1}}}\left(u_{2} x\right) S_{n-i, w^{u_{2}}}^{q^{u_{2}}}\left(u_{1}-1\right) u_{1}^{i-1} u_{2}^{n-i}\right) \frac{t^{n}}{n !} .
\end{aligned}
$$

By the symmetry of $p$-adic invariant integral on $\mathbb{Z}_{p}$, we also see that

$$
\begin{aligned}
T_{w}\left(u_{1}, u_{2} ; x, t\right) & =\left(\frac{1}{u_{2}} \int_{\mathbb{Z}_{p}} w^{u_{2} x_{2}} q^{u_{2} x_{2}} e^{u_{2}\left(x_{2}+u_{1} x\right) t} d x_{2}\right)\left(\frac{u_{2} \int_{\mathbb{Z}_{p}} w^{u_{1} x_{1}} q^{u_{1} x_{1}} e^{u_{1} x_{1} t}}{\int_{\mathbb{Z}_{p}} w^{u_{1} u_{2} x} q^{u_{1} u_{2} x} e^{u_{1} u_{2} x t} d x}\right) \\
& =\sum_{n=0}^{\infty}\left(\sum_{i=0}^{n}\left(\begin{array}{c}
n \\
i
\end{array}\right) B_{i, w^{u_{2}}}^{q^{u_{2}}}\left(u_{1} x\right) S_{n-i, w^{u_{1}}}^{q^{u_{1}}}\left(u_{2}-1\right) u_{2}^{i-1} u_{1}^{n-i}\right) \frac{t^{n}}{n !} .
\end{aligned}
$$

By comparing the coefficients of $t^{n} / n$ ! on both sides of (2.16) and (2.17), we obtain the following theorem.

Theorem 2.1. Let $u_{1}, u_{2}, n \in \mathbb{N}$. Then for all $x \in \mathbb{Z}_{p}$,

$$
\sum_{i=0}^{n}\left(\begin{array}{c}
n \\
i
\end{array}\right) B_{i, w^{u_{1}}}^{q^{u_{1}}}\left(u_{2} x\right) S_{n-i, w^{u_{2}}}^{q^{u_{2}}}\left(u_{1}-1\right) u_{1}^{i-1} u_{2}^{n-i}=\sum_{i=0}^{n}\left(\begin{array}{c}
n \\
i
\end{array}\right) B_{i, w^{u_{2}}}^{q^{u_{2}}}\left(u_{1} x\right) S_{n-i, w^{u_{1}}}^{q^{u_{1}}}\left(u_{2}-1\right) u_{2}^{i-1} u_{1}^{n-i}
$$

where $\left(\begin{array}{c}n \\ i\end{array}\right)$ is the binomial coefficient.

From Theorem 2.1, if we take $u_{2}=1$, then we have the following corollary.

Corollary 2.2. For $m \geq 0$, one we has

$$
B_{i, w}^{q}\left(u_{1} x\right)=\sum_{i=0}^{n}\left(\begin{array}{c}
n \\
i
\end{array}\right) B_{i, w^{u_{1}}}^{q^{u_{1}}}(x) S_{n-i, w}^{q}\left(u_{1}-1\right) u_{1}^{i-1}
$$

where $\left(\begin{array}{c}n \\ i\end{array}\right)$ is the binomial coefficient. 
By (2.17), (2.18), and (2.19), we can see that

$$
\begin{aligned}
T_{w}\left(u_{1}, u_{2} ; x, t\right) & =\left(\frac{e^{u_{1} u_{2} x t}}{u_{1}} \int_{\mathbb{Z}_{p}} w^{u_{1} x} q^{u_{1} x_{1}} e^{u_{1} x_{1} t} d x_{1}\right)\left(\frac{u_{1} \int_{\mathbb{Z}_{p}} w^{u_{2} x_{2}} q^{u_{2} x_{2}} e^{u_{2} x_{2} t} d x_{2}}{\int_{\mathbb{Z}_{p}} w^{u_{1} u_{2} x} q^{u_{1} u_{2} x} e^{u_{1} u_{2} x t} d x}\right) \\
& =\left(\frac{e^{u_{1} u_{2} x t}}{u_{1}} \int_{\mathbb{Z}_{p}} w^{u_{1} x} q^{u_{1} x_{1}} e^{u_{1} x_{1} t} d x_{1}\right)\left(\sum_{i=0}^{u_{1}-1} w^{u_{2} i} q^{u_{2} i} e^{u_{2} i t}\right) \\
& =\frac{1}{u_{1}} \sum_{i=0}^{u_{1}-1} w^{u_{2} i} q^{u_{2} i} \int_{\mathbb{Z}_{p}} w^{u_{1} x} q^{u_{1} x} e^{\left(x_{1}+u_{2} x+\left(u_{2} / u_{1}\right) i\right) t u_{1}} d x_{1} \\
& =\sum_{n=0}^{\infty} \sum_{i=0}^{u_{1}-1} B_{n, w^{u_{1}}}^{q^{u_{1}}}\left(u_{2} x+\frac{u_{2}}{u_{1}} i\right) u_{1}^{n-1} w^{u_{2} i} q^{u_{2} i} \frac{t^{n}}{n !} .
\end{aligned}
$$

From the symmetry of $T_{w}\left(u_{1}, u_{2} ; x, t\right)$, we can also derive

$$
T_{w}\left(u_{1}, u_{2} ; x, t\right)=\sum_{n=0}^{\infty} \sum_{i=0}^{u_{2}-1} B_{n, w^{u_{2}}}^{q^{u_{2}}}\left(u_{1} x+\frac{u_{1}}{u_{2}} i\right) u_{2}^{n-1} w^{u_{1} i} q^{u_{1} i} \frac{t^{n}}{n !}
$$

By comparing the coefficients of $t^{n} / n$ ! on both sides of (2.20) and (2.21), we obtain the following theorem.

Theorem 2.3. For $m \in \mathbb{Z}_{+}, u_{1}, u_{2} \in \mathbb{N}$, we have

$$
\sum_{i=0}^{u_{1}-1} B_{n, w^{u_{1}}}^{q^{u_{1}}}\left(u_{2} x+\frac{u_{2}}{u_{1}} i\right) u_{1}^{n-1} w^{u_{2} i} q^{u_{2} i}=\sum_{i=0}^{u_{2}-1} B_{n, w^{u_{2}}}^{q^{u_{2}}}\left(u_{1} x+\frac{u_{1}}{u_{2}} i\right) u_{2}^{n-1} w^{u_{1} i} q^{u_{1} i}
$$

We note that by setting $u_{2}=1$ in Theorem 2.3, we get the following multiplication theorem for the twisted $q$-Bernoulli polynomials.

Theorem 2.4. For $m \in \mathbb{Z}_{+}, u_{1} \in \mathbb{N}$, one has

$$
B_{n, w}^{q}\left(u_{1} x\right)=u_{1}^{n-1} \sum_{i=0}^{u_{1}-1} B_{n, w^{u_{1}}}^{q^{u_{1}}}\left(x+\frac{i}{u_{1}}\right) w^{i} q^{i}
$$

Remark 2.5. [18], Kim suggested open questions related to finding symmetric properties for Carlitz $q$-Bernoulli numbers. In this paper, we give the symmetric property for $q$-Bernoulli numbers in the viewpoint to give the answer of Kim's open questions.

\section{The Twisted Generalized Bernoulli Polynomials Attached to $x$ of Higher Order}

In this section, we consider the generalized Bernoulli numbers and polynomials and then define the twisted generalized Bernoulli polynomials attached to $x$ of higher order by using 
multivariate $p$-adic invariant integrals on $\mathbb{Z}_{p}$. Let $\chi$ be Dirichlet's character with conductor $d \in \mathbb{N}$. Then the generalized Bernoulli numbers $B_{n, x}$ and polynomials $B_{n, x}(x)$ attached to $\chi$ are defined as

$$
\begin{gathered}
\frac{t \sum_{a=0}^{d-1} X(a) e^{a t}}{e^{d t}-1}=\sum_{n=0}^{\infty} B_{n, X} \frac{t^{n}}{n !}, \\
\frac{t \sum_{a=0}^{d-1} X(a) e^{a t}}{e^{d t}-1} e^{x t}=\sum_{n=0}^{\infty} B_{n, X}(x) \frac{t^{n}}{n !}
\end{gathered}
$$

(cf. $[2,18,23,27])$.

Let $C_{p^{\infty}}=\bigcup_{n \geq 1} C_{p^{n}}=\lim _{n \rightarrow \infty} C_{p^{n}}$ be the locally constant space, where $C_{p^{n}}=\left\{w \mid w^{p^{n}}=\right.$ $1\}$ is the cyclic group of order $p^{n}$. For $w \in C_{p^{\infty}}$, we denote the locally constant function by

$$
\phi_{w}: \mathbb{Z}_{p} \longrightarrow \mathbb{C}_{p}, \quad x \longrightarrow w^{x}
$$

(cf. $[2,3,21,23,24])$. If we take $f(x)=x(x) e^{t x} \phi_{w}(x) q^{x}$, for $q \in \mathbb{C}_{p}$ with $|q-1|_{p}<1$, then it is obvious from (3.1) that

$$
\int_{X} x(x) e^{t x} w^{x} q^{x} d x=\frac{(t+\log q) \sum_{a=0}^{d-1} X(a) w^{a} q^{a} e^{a t}}{w^{d} q^{d} e^{d t}-1}
$$

Now we define the twisted generalized Bernoulli numbers $B_{n, x, w}^{q}$ and polynomials $B_{n, x, w}^{q}(x)$ attached to $X$ as follows:

$$
\begin{gathered}
\frac{(t+\log q) \sum_{a=0}^{d-1} X(a) w^{a} q^{a} e^{a t}}{w^{d} q^{d} e^{d t}-1}=\sum_{n=0}^{\infty} B_{n, x, w}^{q} \frac{t^{n}}{n !} \\
\frac{(t+\log q) \sum_{a=0}^{d-1} X(a) w^{a} q^{a} e^{a t} e^{x t}}{w^{d} q^{d} e^{d t}-1}=\sum_{n=0}^{\infty} B_{n, x, w}^{q}(x) \frac{t^{n}}{n !}
\end{gathered}
$$

for each $w \in C_{p^{\infty}}$ (see [31, 32]). By (3.5) and (3.6),

$$
\begin{gathered}
\int_{X} x(x) x^{n} w^{x} q^{x} d x=B_{n, x, w}^{q} \\
\int_{X} x(y)(x+y)^{n} w^{y} q^{y} d y=B_{n, x, w}^{q}(x) .
\end{gathered}
$$


Thus we have

$$
\begin{aligned}
& \frac{1}{\log q+t}\left(\int_{X} x(x) e^{(n d+x) t} w^{n+x} q^{n+x} d x-\int_{X} x(x) e^{x t} w^{x} q^{x} d x\right) \\
& =\frac{n d \int_{X} X(x) e^{x t} w^{x} q^{x} d x}{\int_{X} e^{n d x t} w^{n d x} q^{n d x} d x} \\
& =\frac{w^{n d} q^{n d} e^{n d t}-1}{w^{d} q^{d} e^{d t}-1} \sum_{i=0}^{d-1} X(i) e^{i t} w^{i} q^{i} .
\end{aligned}
$$

Then

$$
\begin{gathered}
\frac{1}{\log q+t}\left(\int_{X} x(x) e^{(n d+x) t} w^{n+x} q^{n+x} d x-\int_{X} x(x) e^{x t} w^{x} q^{x} d x\right) \\
=\sum_{l=0}^{n d-1} x(l) e^{l t} w^{l} q^{l}=\sum_{k=0}^{\infty} \sum_{l=0}^{n d-1} x(l) l^{k} w^{l} q^{l} \frac{t^{k}}{k !} .
\end{gathered}
$$

Let us define the $p$-adic twisted $q$-function $T_{k, w}^{q}(\chi, n)$ as follows:

$$
T_{k, w}^{q}(x, n)=\sum_{l=0}^{n} \chi(l) l^{k} w^{l} q^{l}
$$

By (3.9) and (3.10), we see that

$$
\frac{1}{\log q+t}\left(\int_{X} x(x) e^{(n d+x) t} w^{n d+x} q^{n d+x} d x-\int_{X} x(x) e^{x t} w^{x} q^{x} d x\right)=\sum_{k=0}^{\infty} T_{k, w}^{q}(x, n d-1) \frac{t^{k}}{k !}
$$

Thus,

$$
\left(\int_{X} x(x)(n d+x)^{k} w^{n+x} q^{n+x} d x-\int_{X} x(x) x^{k} w^{x} q^{x} d x\right)=(t+\log q) T_{k, w}^{q}(x, n d-1),
$$

for all $k, n, d \in \mathbb{N}$. This means that

$$
B_{k, x, w}^{q}(n d)-B_{n, x, w}^{q}=(t+\log q) T_{k, w}^{q}(x, n d-1),
$$


for all $k, n, d \in \mathbb{N}$. For all $u_{1}, u_{2}, d \in \mathbb{N}$, we have

$$
\begin{aligned}
& \frac{d \int_{X} \int_{X} X\left(x_{1}\right) X\left(x_{2}\right) e^{\left(w_{1} x_{1}+w_{2} x_{2}\right) t} w^{u_{1} x_{1}+u_{2} x_{2}} q^{u_{1} x_{1}+u_{2} x_{2}} d x_{1} d x_{2}}{\int_{X} e^{d u_{1} u_{2} x t} w^{d u_{1} u_{2} x} q^{d u_{1} u_{2} x} d x} \\
& =\frac{(t+\log q)\left(e^{d u_{1} u_{2} t} w^{d u_{1} u_{2}} q^{d u_{1} u_{2}}-1\right)}{\left(e^{d u_{1} t} w^{d u_{1}} q^{d u_{1}}-1\right)\left(e^{d u_{2} t} w^{d u_{2}} q^{d u_{2}}-1\right)} \\
& \quad \times\left(\sum_{a=0}^{d-1} X(a) e^{u_{1} a t} w^{u_{1} a} q^{u_{1} a}\right)\left(\sum_{b=0}^{d-1} X(b) e^{u_{2} b t} w^{u_{2} b} q^{u_{2} b}\right) .
\end{aligned}
$$

The twisted generalized Bernoulli numbers $B_{n, x, w}^{(k, q)}$ and polynomials $B_{n, x, w}^{(k, q)}(x)$ attached to $x$ of order $k$ are defined as

$$
\begin{gathered}
\left(\frac{(t+\log q) \sum_{a=0}^{d-1} X(a) w^{a} q^{a} e^{a t}}{w^{d} q^{d} e^{d t}-1}\right)^{k}=\sum_{n=0}^{\infty} B_{n, x, w}^{(k, q)} \frac{t^{n}}{n !} \\
\left(\frac{(t+\log q) \sum_{a=0}^{d-1} X(a) w^{a} q^{a} e^{a t}}{w^{d} q^{d} e^{d t}-1}\right)^{k} e^{x t}=\sum_{n=0}^{\infty} B_{n, x, w}^{(k, q)}(x) \frac{t^{n}}{n !}
\end{gathered}
$$

for each $w \in C_{p^{\infty}}$. For $u_{1}, u_{2} \in \mathbb{N}$, we set

$$
\begin{aligned}
K_{w}^{q}\left(m, x ; u_{1}, u_{2}\right) & \\
= & \frac{d \int_{X^{m}} \prod_{i=1}^{m} X\left(x_{i}\right) e^{\left(\sum_{i=1}^{m} x_{i}+u_{2} x\right) u_{1} t} w^{\left(\sum_{i=1}^{m} x_{i}+u_{2} x\right) u_{1}} q^{\left(\sum_{i=1}^{m} x_{i}+u_{2} x\right) u_{1}} d x_{1} \cdots d x_{m}}{\int_{X} e^{d u_{1} u_{2} x t} w^{d u_{1} u_{2} x} q^{d u_{1} u_{2} x} d x} \\
& \times \int_{X^{m}} \prod_{i=1}^{m} X\left(x_{i}\right) e^{\left(\sum_{i=1}^{m} x_{i}+u_{1} y\right) u_{2} t} w^{\left(\sum_{i=1}^{m} x_{i}+u_{1} y\right) u_{2}} q^{\left(\sum_{i=1}^{m} x_{i}+u_{1} y\right) u_{1}} d x_{1} \cdots d x_{m},
\end{aligned}
$$

where $\int_{X^{m}} f\left(x_{1} \cdots x_{m}\right) d x_{1} \cdots d x_{m}=\int_{X} \cdots \int_{X} f\left(x_{1}, \ldots, x_{m}\right) d x_{1} \cdots d x_{m}$. In (3.17), we note that $\mathrm{K}_{w}^{q}\left(m, \chi ; u_{1}, u_{2}\right)$ is symmetric in $u_{1}, u_{2}$. From (3.17), we have

$$
\begin{aligned}
& K_{w}^{q}\left(m, \chi ; u_{1}, u_{2}\right)=\int_{X^{m}} \prod_{i=1}^{m} \chi\left(x_{i}\right) e^{\left(\sum_{i=1}^{m} x_{i}\right) u_{2} t} w^{\left(\sum_{i=1}^{m} x_{i}\right) u_{2}} q^{\left(\sum_{i=1}^{m} x_{i}\right) u_{2}} d x_{1} \cdots d x_{m} \\
& \times e^{u_{1} u_{2} x t} w^{u_{1} u_{2} x} q^{u_{1} u_{2} x}\left(\frac{d \int_{X} X\left(x_{m}\right) e^{u_{2} x_{m} t} w^{u_{2} x_{m}} q^{u_{2} x_{m}} d x_{m}}{\int_{X} e^{d u_{1} u_{2} x} q^{d u_{1} u_{2} x} d x}\right) \\
& \times \int_{X^{m-1}} \prod_{i=1}^{m-1} \chi\left(x_{i}\right) e^{\left(\sum_{i=1}^{m-1} x_{i}\right) u_{2} t} w^{\left(\sum_{i=1}^{m-1} x_{i}\right) u_{2}} q^{\left(\sum_{i=1}^{m-1} x_{i}\right) u_{2}} d x_{1} \cdots d x_{m-1} \\
& \times e^{u_{1} u_{2} y t} w^{u_{1} u_{2} y} q^{u_{1} u_{2} y} .
\end{aligned}
$$


Thus we can obtain

$$
\begin{gathered}
\frac{u_{1} d \int_{X} X(x) e^{x t} w^{x} q^{x} d x}{\int_{X} e^{d u_{2} x t} w^{d u_{2} x} q^{d u_{2} x} d x}=\sum_{k=0}^{\infty}\left(\sum_{i=0}^{u_{1} d-1} x(i) i^{k} w^{i} q^{i}\right) \frac{t^{k}}{k !}=\sum_{k=0}^{\infty} T_{k, w}^{q}\left(x, u_{1} d-1\right) \frac{t^{k}}{k !} \\
e^{u_{1} u_{2} x t} w^{u_{1} u_{2} x} q^{u_{1} u_{2} x} \int_{X^{m}} \prod_{i=1}^{m} x\left(x_{i}\right) e^{\left(\sum_{i=1}^{m} x_{i}\right) u_{1} t} w^{\left(\sum_{i=1}^{m} x_{i}\right) u_{1}} q^{\left(\sum_{i=1}^{m} x_{i}\right) u_{1}} d x_{1} \cdots d x_{m} \\
=e^{u_{1} u_{2} x t} w^{u_{1} u_{2} x} q^{u_{1} u_{2} x}\left(\frac{u_{1}}{e^{d u_{1} t} w^{d u_{1}} q^{d u_{1}}-1} \sum_{a=0}^{d-1} X(a) e^{u_{1} a t} w^{u_{1} a} q^{u_{1} a}\right) \\
=\sum_{n=0}^{\infty} B_{n, x, w}^{(m, q)}\left(u_{2} x\right) u_{1}^{n} \frac{t^{n}}{n !}
\end{gathered}
$$

From (3.19), we derive

$$
\begin{aligned}
K_{w}^{q}( & \left.m, x ; u_{1}, u_{2}\right) \\
\quad= & \sum_{l=0}^{\infty} B_{l, x, w}^{(m, q)}\left(u_{1} x\right) u_{1}^{l} \frac{t^{l}}{l !} \sum_{k=0}^{\infty} T_{k, w}^{q}\left(x, u_{1} d-1\right) \frac{t^{k}}{k !}\left(\sum_{i=0}^{\infty} B_{i, x, w}^{(m-1, q)}\left(u_{1} y\right) \frac{u_{2}^{i} t^{i}}{i !}\right) \frac{1}{u_{1}} \\
& =\sum_{n=0}^{\infty} \sum_{j=0}^{n}\left(\begin{array}{c}
n \\
j
\end{array}\right) u_{2}^{j} u_{1}^{n-j-1} B_{n-j, x, w}^{(m, q)}\left(u_{2} x\right) \times \sum_{k=0}^{j} T_{k, w}^{q}\left(x, u_{1} d-1\right)\left(\begin{array}{c}
j \\
k
\end{array}\right) B_{j-k, x, w}^{(m-1, q)}\left(u_{1} y\right) \frac{t^{n}}{n !} .
\end{aligned}
$$

By the symmetry of $K_{w}^{q}\left(m, x ; u_{1}, u_{2}\right)$ in $u_{1}$ and $u_{2}$, we can see that

$$
\begin{aligned}
K_{w}^{q}\left(m, x ; u_{1}, u_{2}\right) \\
\quad=\sum_{n=0}^{\infty} \sum_{j=0}^{n}\left(\begin{array}{c}
n \\
j
\end{array}\right) u_{1}^{j} u_{2}^{n-j-1} B_{n-j, x, w}^{(m, q)}\left(u_{1} x\right) \times \sum_{k=0}^{j} T_{k, w}^{q}\left(x, u_{2} d-1\right)\left(\begin{array}{c}
j \\
k
\end{array}\right) B_{j-k, x, w}^{(m-1, q)}\left(u_{2} y\right) \frac{t^{n}}{n !} .
\end{aligned}
$$

By comparing the coefficients on both sides of (3.20) and (3.21), we see the following theorem.

Theorem 3.1. For $d, u_{1}, u_{2}, m \in \mathbb{N}, n \in \mathbb{Z}$, one has

$$
\begin{aligned}
& \sum_{j=0}^{n}\left(\begin{array}{c}
n \\
j
\end{array}\right) u_{2}^{j} u_{1}^{n-j-1} B_{n-j, x, w}^{(m, q)}\left(u_{2} x\right) \sum_{k=0}^{j} T_{k, w}^{q}\left(x, u_{1} d-1\right)\left(\begin{array}{l}
j \\
k
\end{array}\right) B_{j-k, x, w}^{(m-1, q)}\left(u_{1} y\right) \\
& =\sum_{j=0}^{n}\left(\begin{array}{c}
n \\
j
\end{array}\right) u_{1}^{j} u_{2}^{n-j-1} B_{n-j, x, w}^{(m, q)}\left(u_{1} x\right) \sum_{k=0}^{j} T_{k, w}^{q}\left(x, u_{2} d-1\right)\left(\begin{array}{l}
j \\
k
\end{array}\right) B_{j-k, x, w}^{(m-1, q)}\left(u_{2} y\right) .
\end{aligned}
$$


Remark 3.2. If we take $y=0$ and $m=1$ in (3.22), then we have

$$
\begin{aligned}
& \sum_{j=0}^{n}\left(\begin{array}{l}
n \\
j
\end{array}\right) u_{2}^{j} u_{1}^{n-j-1} B_{n-j, x, w}^{q}\left(u_{2} x\right) \sum_{k=0}^{j} T_{k, w}^{q}\left(x, u_{1} d-1\right)\left(\begin{array}{l}
j \\
k
\end{array}\right) \\
& =\sum_{j=0}^{n}\left(\begin{array}{l}
n \\
j
\end{array}\right) u_{1}^{j} u_{2}^{n-j-1} B_{n-j, x, w}^{q}\left(u_{1} x\right) \sum_{k=0}^{j} T_{k, w}^{q}\left(x, u_{2} d-1\right)\left(\begin{array}{l}
j \\
k
\end{array}\right) .
\end{aligned}
$$

Now we can also calculate

$$
K_{w}^{q}\left(m, x ; u_{1}, u_{2}\right)=\sum_{n=0}^{\infty}\left(\sum_{k=0}^{n}\left(\begin{array}{l}
n \\
k
\end{array}\right) u_{1}^{k-1} u_{2}^{n-k} B_{n-k, x, w}^{(m-1, q)}\left(u_{1} y\right) \sum_{i=0}^{d u_{1}-1} B_{i, x, w}^{(m, q)}\left(u_{2} x+\frac{u_{2}}{u_{1}} i\right)\right) \frac{t^{n}}{n !} .
$$

From the symmetric property of $K_{w}^{q}\left(m, x ; u_{1}, u_{2}\right)$ in $u_{1}$ and $u_{2}$, we derive

$$
K_{w}^{q}\left(m, x ; u_{1}, u_{2}\right)=\sum_{n=0}^{\infty}\left(\sum_{k=0}^{n}\left(\begin{array}{l}
n \\
k
\end{array}\right) u_{2}^{k-1} u_{1}^{n-k} B_{n-k, x, w}^{(m-1, q)}\left(u_{2} y\right) \sum_{i=0}^{d u_{2}-1} B_{i, x, w}^{(m, q)}\left(u_{1} x+\frac{u_{1}}{u_{2}} i\right)\right) \frac{t^{n}}{n !} .
$$

By comparing the coefficients on both sides of (3.24) and (3.26), we obtain the following theorem.

Theorem 3.3. For $d, u_{1}, u_{2}, m \in \mathbb{N}, n \in \mathbb{Z}$, we have

$$
\begin{aligned}
\sum_{k=0}^{n}\left(\begin{array}{l}
n \\
k
\end{array}\right) u_{1}^{k-1} u_{2}^{n-k} B_{n-k, x, w}^{(m-1, q)}\left(u_{1} y\right) \sum_{i=0}^{d u_{1}-1} B_{k, x, w}^{(m, q)}\left(u_{2} x+\frac{u_{2}}{u_{1}} i\right) \\
=\sum_{k=0}^{n}\left(\begin{array}{l}
n \\
k
\end{array}\right) u_{2}^{k-1} u_{1}^{n-k} B_{n-k, x, w}^{(m-1, q)}\left(u_{2} y\right) \sum_{i=0}^{d u_{2}-1} B_{k, x, w}^{(m, q)}\left(u_{1} x+\frac{u_{1}}{u_{2}} i\right) .
\end{aligned}
$$

Remark 3.4. If we take $y=0$ and $m=1$ in (3.26), then one has

$$
u_{1}^{n-1} \sum_{i=0}^{d u_{1}-1} B_{n, x, w}^{q}\left(u_{2} x+\frac{u_{2}}{u_{1}} i\right)=u_{2}^{n-1} \sum_{i=0}^{d u_{2}-1} B_{n, x, w}^{q}\left(u_{1} x+\frac{u_{1}}{u_{2}} i\right) .
$$

Remark 3.5. In our results for $q=1$, we can also derive similar results, which were treated in [27]. In this paper, we used the $p$-adic integrals to derive the symmetric properties of the $q$-Bernoulli polynomials. By using the symmetric properties of $p$-adic integral on $X$, we can easily derive many interesting symmetric properties related to Bernoulli numbers and polynomials. 


\section{Acknowledgments}

The authors express Their sincere gratitude to referees for their valuable suggestions and comments. This work has been conducted by the Research Grant of Kwangwoon University in 2010.

\section{References}

[1] M. Cenkci, Y. Simsek, and V. Kurt, "Further remarks on multiple $p$-adic $q$ - $L$-function of two variables," Advanced Studies in Contemporary Mathematics, vol. 14, no. 1, pp. 49-68, 2007.

[2] L.-C. Jang, "On a $q$-analogue of the $p$-adic generalized twisted $L$-functions and $p$-adic $q$-integrals," Journal of the Korean Mathematical Society, vol. 44, no. 1, pp. 1-10, 2007.

[3] L.-C. Jang, "Multiple twisted $q$-Euler numbers and polynomials associated with $p$-adic $q$-integrals," Advances in Difference Equations, vol. 2008, Article ID 738603, 11 pages, 2008.

[4] L.-C. Jang, S.-D. Kim, D.-W. Park, and Y.-S. Ro, “A note on Euler number and polynomials," Journal of Inequalities and Applications, vol. 2006, Article ID 34602, 5 pages, 2006.

[5] L.-C. Jang and T. Kim, "On the distribution of the $q$-Euler polynomials and the $q$-Genocchi polynomials of higher order," Journal of Inequalities and Applications, vol. 2008, Article ID 723615, 9 pages, 2008.

[6] T. Kim, "q-Volkenborn integration," Russian Journal of Mathematical Physics, vol. 9, no. 3, pp. 288-299, 2002.

[7] T. Kim, “On Euler-Barnes multiple zeta functions," Russian Journal of Mathematical Physics, vol. 10, no. 3, pp. 261-267, 2003.

[8] T. Kim, "Analytic continuation of multiple $q$-zeta functions and their values at negative integers," Russian Journal of Mathematical Physics, vol. 11, no. 1, pp. 71-76, 2004.

[9] T. Kim, "Power series and asymptotic series associated with the $q$-analog of the two-variable $p$-adic L-function," Russian Journal of Mathematical Physics, vol. 12, no. 2, pp. 186-196, 2005.

[10] T. Kim, "Multiple p-adic L-function," Russian Journal of Mathematical Physics, vol. 13, no. 2, pp. 151$157,2006$.

[11] T. Kim, "A note on $p$-Adic $q$-integral on Zp associated with $q$-Euler numbers," Advanced Studies in Contemporary Mathematics, vol. 15, pp. 133-138, 2007.

[12] T. Kim, "On $p$-adic interpolating function for $q$-Euler numbers and its derivatives," Journal of Mathematical Analysis and Applications, vol. 339, no. 1, pp. 598-608, 2008.

[13] T. Kim, "On the analogs of Euler numbers and polynomials associated with $p$-adic $q$-integral on $\mathbb{Z}_{p}$ at $q=-1, "$ Journal of Mathematical Analysis and Applications, vol. 331, no. 2, pp. 779-792, 2007.

[14] T. Kim, "A note on $p$-adic $q$-integral on $\mathbb{Z}_{p}$ associated with $q$-Euler numbers," Advanced Studies in Contemporary Mathematics, vol. 15, no. 2, pp. 133-137, 2007.

[15] T. Kim, " $q$-Euler numbers and polynomials associated with $p$-adic $q$-integrals," Journal of Nonlinear Mathematical Physics, vol. 14, no. 1, pp. 15-27, 2007.

[16] T. Kim, "A note on some formulae for the q-Euler numbers and polynomials," Proc. Jangjeon Math. Soc., vol. 9, no. 2, pp. 227-232, 2006.

[17] T. Kim, " $q$-Bernoulli numbers and polynomials associated with Gaussian binomial coefficients," Russian Journal of Mathematical Physics, vol. 15, no. 1, pp. 51-57, 2008.

[18] T. Kim, "On the symmetries of the $q$-Bernoulli polynomials," Abstract and Applied Analysis, vol. 2008, Article ID 914367, 7 pages, 2008.

[19] T. Kim, "Note on Dedekind type DC sums," Advanced Studies in Contemporary Mathematics, vol. 18, no. 2, pp. 249-260, 2009.

[20] T. Kim, L.-C. Jang, and H. K. Pak, "A note on q-Euler and Genocchi numbers," Proceedings of the Japan Academy, Series A, vol. 77, no. 8, pp. 139-141, 2001.

[21] T. Kim, "Note on the $q$-Euler numbers of higher order," Advanced Studies in Contemporary Mathematics, vol. 19, no. 1, pp. 25-29, 2009.

[22] T. Kim, M.-S. Kim, L.-C. Jang, and S.-H. Rim, “New q-Euler numbers and polynomials associated with p-adic q-integrals," Advanced Studies in Contemporary Mathematics, vol. 15, no. 2, pp. 243-252, 2007.

[23] W. Kim, Y.-H. Kim, and L.-C. Jang, "On the q-extension of apostol-euler numbers and polynomials," Abstract and Applied Analysis, vol. 2008, Article ID 296159, 10 pages, 2008. 
[24] Y. Simsek, "Generating functions of the twisted Bernoulli numbers and polynomials associated with their interpolation functions," Advanced Studies in Contemporary Mathematics, vol. 16, no. 2, pp. 251$278,2008$.

[25] H. Ozden, Y. Simsek, S.-H. Rim, and I. N. Cangul, "A note on $p$-adic $q$-Euler measure," Advanced Studies in Contemporary Mathematics, vol. 14, pp. 233-239, 2007.

[26] S.-H. Rim, Y.-H. Kim, B. J. Lee, and T. Kim, "Some identities of the generalized twisted Bernoulli numbers and polynomials of higher order," Journal of Computational Analysis and Applications, vol. 12, pp. 695-702, 2010.

[27] T. Kim, "On a $q$-analogue of the $p$-adic log gamma functions and related integrals," Journal of Number Theory, vol. 76, no. 2, pp. 320-329, 1999.

[28] T. Kim, “Note on the Euler q-zeta functions," Journal of Number Theory, vol. 129, no. 7, pp. 1798-1804, 2009.

[29] T. Kim, "A new approach to $p$-adic $q$-L-functions," Advanced Studies in Contemporary Mathematics, vol. 12, no. 1, pp. 61-72, 2006.

[30] T. Kim and S.-H. Rim, "On the twisted $q$-Euler numbers and polynomials associated with basic $q$ - $l$ functions," Journal of Mathematical Analysis and Applications, vol. 336, no. 1, pp. 738-744, 2007.

[31] T. Kim, "New approach to q-Euler polynomials of higher order," Russian Journal of Mathematical Physics, vol. 17, no. 2, pp. 201-207, 2010.

[32] T. Kim, "Barnes-type multiple $q$-zeta functions and $q$-Euler polynomials," Journal of Physics A, vol. 43, Article ID 255201, 11 pages, 2010. 